cochlear implants. Three quarters of the cases were detected at routine follow-up after meningitis. Risk factors for hearing loss were as follows: symptoms of meningitis for $>2$ days before admission, absence of petechiae, CSF glucose $<0.6 \mathrm{mmol} / \mathrm{L}$, Streptococcus pneumoniae, and ataxia. Based on this prediction rule of 5 factors, $62 \%$ of postmeningitic children were considered at risk. All cases of hearing loss were in this at-risk group. (Koomen I, Grobbee DE, Roord JJ, et al. Hearing loss at school age in survivors of bacterial meningitis: Assessment, incidence, and prediction. Pediatrics November 2003;112:1049-1053). (Reprints: AM van Furth MD, PhD, VU Medical Center, Department of Pediatrics, Box 7057, 1007 MB Amsterdam, the Netherlands).

COMMENT. The inclusion of hearing evaluation in the routine follow-up of children after bacterial meningitis should prevent the missed diagnoses of hearing loss. The occurrence of prediction factors should mandate repeated hearing tests to rule out fluctuating or late onset hearing loss. Vaccination against $S$ pneumoniae in children aged 2 years or older with certain risk factors (AAP Red Book 2000) may prevent some cases and may alter the prediction rule established in this study.

The omission of some important references from the current report is unfortunate. A seminal study and prospective evaluation of hearing impairment as a sequel of acute bacterial meningitis was reported by Dodge $\mathrm{PR}$ and colleagues ( $\mathrm{N}$ Engl J Med 1984;311:869).. Post-meningitic sensorineural hearing loss pre-1980 occurred in up to $30 \%$ of patients with pneumococcal meningitis, $10 \%$ of meningococcal, and $5-20 \%$ of $\mathrm{H}$ Influenzae type B meningitis. Regardless of the bacteria, type of antibiotic therapy, or use of dextromethasone, audiological assessment was recommended in all meningitis patients before or soon after discharge from hospital.

Another report emphasized psychological and educational adverse outcomes as well as deafness in a group of 130 children examined at a mean age of 8 years, and 6 years after meningitis (Grimwood K, et al. Pediatrics 1995;95:646-656; see Ped Neur Briefs June 1995). In this Australian study, even with optimal therapy, one in 4 children who recovered from meningitis had severe or functionally significant disabilities that affected school performance.

\title{
ABSCESS OF CAVUM SEPTUM PELLUCIDUM COMPLICATING PNEUMOCOCCAL MENINGITIS
}

A 7-year-old boy with pneumococcal meningitis complicated by abscesses involving the cavum septum pellucidum (CSP) and cavum vergae (CV) is reported from the Children's Hospital and University of California San Diego, CA. He was admitted with fever, headache, vomiting, and nuchal rigidity. Symptoms of rhinorrhea and cough had developed 9 days before admission. CT scan demonstrated sinusitis, left frontal subdural empyema, and abscesses of the CSP and CV. Streptococcus pneumoniae was isolated from the CSF and was susceptible to penicillin and ceftriaxone. Antibiotic and dexamethasone therapy resulted in resolution of fever, headache, and nuchal rigidity. Relapse occurred 3 days after discharge, and CT showed an interval increase in size of CSP and CV. Vancomycin was added to the ceftriaxone and the CSF culture was sterile. A repeat CT 5 days later showed further increase in size of CSP and CV, and hydrocephalus. CT-guided drainage of the CSP released $10 \mathrm{ml}$ of purulent material with negative culture. Following 6 
weeks of ceftriaxone and 4 weeks of added vancomycin therapy, CT demonstrated resolution of the CSP abscess and mild enhancement of the CV. The patient recovered with no sequelae. (Pong A, James HE, Senac MO, Jr, et al. Pneumococcal infection of the cavum septi pellucidi and cavum vergae in a pediatric patient. Pediatr Infect Dis J November 2003;22:1014-1017). (Respond: Alice Pong MD, Division of Pediatric Infectious Diseases, Children's Hospital and Health Center, University of California, San Diego, CA).

COMMENT. CSP and CV are a normal finding in infants less than 6 months, but are usually closed by 6 months of age. They can occur as an incidental finding in $<20 \%$ of children $>6$ months of age. Hydrocephalus may occur if the cysts enlarge and obstruct the foramen of Munro. Congenital intracranial cysts are discussed in McLone DG, ed Pediatric Neurosurgery $4^{\text {th }}$ ed, Philadelphia:Saunders, 2001:489-498. The present report is considered the first case of postneonatal childhood abscess of the CSP and CV with pneumococcal meningitis. Five previous reports of infection of the CSP and CV are cited by the authors, 4 in adults and one in a 31-week gestational age neonate with an abscess of the CSP and hydrocephalus associated with Proteus mirabilis meningitis (Li ST, et al. 2002). The abscess resolved after ventriculoperitoneal shunting.

\section{NEUROMUSCULAR DISORDERS}

\section{EFFECT OF MATERNAL MYASTHENIA ON DELIVERY AND THE NEWBORN}

The effect of pre-existing myasthenia gravis (MG) on delivery and the newborn was investigated in a retrospective study of 127 births by mothers with MG compared to 1.9 million births by mothers without MG at the University of Bergen, Norway. Women with MG have an increased risk of complications at delivery, especially the risk of premature rupture of amniotic membranes ( $5.5 \%$ vs $1.7 \%, \mathrm{p}=0.001)$. Risk of cesarean sections doubled $(17.3 \%$ vs $8.6 \%, \mathrm{p}=0.001)$. Birth defects and/or neonatal complications occurred in $27(21 \%)$ of the 127 children, and $5(3.9 \%)$ had severe defects vs $1.9 \%$ in the reference group (NS). Three with severe defects died. Neonatal MG was diagnosed in 5 cases. Perinatal mortality was $2.4 \%$ in the MG group vs $1.4 \%$ in the reference group $(\mathrm{p}=0.7$, NS). A comparison of 45 births in mothers previously thymectomized and 82 nonthymectomy MG births found no significant differences regarding delivery and birth complications or defects. (Hoff JM, Daltveit AK, Gilhus NE. Myasthenia gravis: Consequences for pregnancy, delivery, and the newborn. Neurology November (2 of 2) 2003;61:1362-1366). (Reprints: Dr Jana Midelfart Hoff, Institute of Neurology, University of Bergen, 5021 Bergen, Norway).

COMMENT. MG is associated with an increased risk of complications during delivery and higher rate of ceasarean sections. A higher rate of birth defects and perinatal mortality in infants born to MG mothers is not significantly different from non-MG births. Prior thymectomy does not prevent the increase in delivery complications in MG mothers. 